\title{
Study Design and Cohort Comparability in a Study of Major Cardiovascular Events in New Users of Prucalopride Versus Polyethylene Glycol 3350
}

\author{
Joan Fortuny ${ }^{1} \cdot$ Alicia Gilsenan $^{2} \cdot$ Miguel Cainzos-Achirica $^{1,7} \cdot$ Oscar F. Cantero $^{3} \cdot$ Robert W. V. Flynn $^{4}$. \\ Luis Garcia-Rodriguez ${ }^{3}$ - Bianca Kollhorst ${ }^{5}$. Pär Karlsson ${ }^{6} \cdot$ Love Linnér $^{6} \cdot$ Thomas M. MacDonald $^{4}$ - Estel Plana ${ }^{1}$. \\ Ana Ruigómez $z^{3} \cdot$ Tania Schink $^{5} \cdot$ Ryan Ziemiecki $^{2} \cdot$ Elizabeth B. Andrews $^{2}$
}

Published online: 27 May 2019

(c) The Author(s) 2019

\begin{abstract}
Introduction Given prior safety experience with other 5- $\mathrm{HT}_{4}$ agonists for chronic constipation, an observational, populationbased cohort study in five data sources from Germany, Sweden, and the UK was conducted to evaluate the cardiovascular safety of prucalopride.

Objectives Our objective is to describe the methods and resulting comparability of cohorts in a multi-database, multinational study of prucalopride initiators and polyethylene glycol 3350 (PEG) initiators following a harmonized protocol.

Methods Prucalopride initiators were matched on age, sex, and index date to PEG initiators (1:5 ratio). Study exposures, cardiovascular risk factors, and other covariates were identified from healthcare utilization codes harmonized across databases. Cardiovascular outcomes were identified using database-specific algorithms based on diagnosis codes. The propensity score (PS) in each database was estimated using logistic regression, with prucalopride versus PEG as the outcome and including clinically relevant variables associated with major adverse cardiovascular events.

Results In total, 12,030 prucalopride initiators and 59,985 PEG initiators were identified. After matching and trimming, cohorts from the UK and Sweden were well-balanced for cardiovascular risk factors and cancer. However, in Germany, PEG initiators remained older and sicker than prucalopride initiators. The prevalence of these characteristics also differed from those in the UK and Sweden. The pooled analyses included only data from the UK and Sweden.

Conclusions Matching, trimming, and PS stratification yielded comparable cohorts in four of five data sources. Use of these methods could not achieve balance for key covariates within the German cohort, likely due to reimbursement differences in Germany.
\end{abstract}

\section{Introduction}

Chronic constipation significantly impairs health-related quality of life and is associated with a considerable economic burden $[1,2]$. The 5 -hydroxytryptamine (serotonin) receptor type $4\left(5-\mathrm{HT}_{4}\right)$ receptor agonists have the potential to treat disorders of motility throughout the gastrointestinal tract, including chronic constipation [3]. The first-generation

Electronic supplementary material The online version of this article (https://doi.org/10.1007/s40264-019-00836-z) contains supplementary material, which is available to authorized users.

Alicia Gilsenan

agilsenan@rti.org

Extended author information available on the last page of the article
5- $\mathrm{HT}_{4}$ receptor agonists, cisapride and tegaserod, are nonselective and interact with other receptors in a concentration range relevant for their interaction with the $5-\mathrm{HT}_{4}$ receptor. Cisapride was withdrawn from the US market in 2000 because of its association with torsade de pointes arrhythmia secondary to QT interval prolongation, and tegaserod was withdrawn from the US market in 2007 because of a possible association with ischemic cardiovascular events.

Prucalopride is a selective 5- $\mathrm{HT}_{4}$ agonist with high affinity and selectivity for the 5- $\mathrm{HT}_{4}$ receptors [4]. Prucalopride has been licensed in the EU since 2009 for adult women and since 2015 for adult men for the treatment of chronic constipation.

Despite the absence of a signal for increased risk of adverse cardiovascular events for prucalopride during nonclinical and clinical cardiovascular investigations and safety 


\section{Key Points}

In this population-based, multinational cohort study to evaluate the cardiovascular safety of prucalopride versus polyethylene glycol 3350 (PEG), use of a common protocol and definitions, as well as propensity score (PS) stratification and trimming, yielded comparable study cohorts in four of the five data sources.

Differences in reimbursement practices in Germany meant that these analytic methods could not achieve balance for key covariates within the German cohort.

Thus, only data from the UK and Sweden (i.e. four of five data sources) were eventually included in the pooled analyses.

monitoring, the US FDA expressed interest in having additional information about cardiovascular safety to support the new drug application of prucalopride [4]. The availability of prucalopride in the EU offered a unique opportunity to conduct an observational pharmacoepidemiological safety study to evaluate its cardiovascular safety in a real-world setting.

In the context of a multinational, multi-database study using a common protocol to evaluate the occurrence of major adverse cardiovascular events (MACE) among initiators of prucalopride and initiators of polyethylene glycol (PEG), the aim of this manuscript is to show the performance of propensity score (PS) stratification and trimming to obtain comparable study cohorts; and to report study design decisions undertaken when balance could not be achieved. The findings in terms of MACE of the prucalopride safety study are reported elsewhere [5].

\section{Methods}

\subsection{Study Design}

This was an observational population-based cohort study of patients initiating prucalopride or PEG in Germany, Sweden, and the UK (including England, Wales, Scotland and Northern Ireland) following a common protocol (EUPAS9200) [6]. PEG was chosen as an indicator of chronic constipation because it was the most frequently used reimbursed medication for chronic constipation and was not thought to be associated with a modified risk of cardiovascular events.

The study used a common protocol and harmonized study materials across data sources (definitions of outcomes and covariates, table structure and analytical approach). The study followed a new-user (initiator) design [7]. Initiators were individuals starting a study medication for the first time who may have used the other study medication in the past, with certain restrictions specified in Sect. 2.3. Where possible, patients were followed until 7 days after the estimated end of use of prucrictions specifiealopride or PEG. The 7-day extension period was chosen based on the terminal half-life of prucalopride (approximately 1 day) [8].

\subsection{Setting}

The study was implemented using five population-based automated healthcare databases. In the UK, two data sources derived from electronic medical records from general practices in England, Wales, and Northern Ireland were used: the Clinical Practice Research Datalink (CPRD), which included linkage to Hospital Episode Statistics (HES) for inpatient data and Office of National Statistics (ONS) data for cause-of-death data for some participating practices; and The Health Improvement Network (THIN). The Information Services Division (ISD) from Scotland, a data source derived from an administrative healthcare database, was also used. In Sweden, the Swedish National Registers (SNR), which include the National Patient Register, the Prescribed Drug Register, the National Cancer Register, the Causeof-Death Register, and the Total Population Register, were used. Finally, in Germany, the German Pharmacoepidemiological Research Database (GePaRD), a claims database of four statutory healthcare insurance providers, was used. All relevant authorities reviewed and approved the study on ethical grounds in the UK (Independent Scientific Advisory Committee for CPRD, Scientific Review Committee for THIN and East of Scotland Research Ethics Services/ Public Benefit and Privacy Panel for Health and Social Care for ISD), Germany (all involved health insurance providers, as well as the German Federal [Social] Insurance Office and the Senator for Science, Health, and Consumer Protection in Bremen), and Sweden (Regional Ethical Review Board, Stockholm). The study period was 2010-2016 in CPRD, THIN, and ISD; 2012-2015 in SNR; and 2010-2014 in GePaRD.

The data sources were chosen based on the knowledge that prucalopride was reimbursed in their respective countries, they had available information on the exposures and outcomes of interest for the study on essential demographic and clinical covariates (see Table 1), and were able and willing to participate in the study. The data sources are described in detail in Electronic Supplementary Material (ESM) 1.

\subsection{Study Population}

Patients with a record of a dispensing (ISD, SNR, GePaRD) or prescription (CPRD, THIN) for prucalopride or PEG were identified retrospectively from existing records (i.e. 
Table 1 Distribution of demographic, cardiovascular, and gastrointestinal covariates after trimming between exposure cohorts

\begin{tabular}{|c|c|c|c|c|c|c|}
\hline \multirow[t]{2}{*}{ Covariate } & \multicolumn{2}{|c|}{ UK (CPRD, THIN, ISD) } & \multicolumn{2}{|l|}{ SNR } & \multicolumn{2}{|l|}{ GePaRD } \\
\hline & $\begin{array}{l}\text { Prucalopride } \\
N=2521\end{array}$ & $\begin{array}{l}\text { PEG } \\
N=12,603\end{array}$ & $\begin{array}{l}\text { Prucalopride } \\
N=3194\end{array}$ & $\begin{array}{l}\text { PEG } \\
N=16,769\end{array}$ & $\begin{array}{l}\text { Prucalopride } \\
N=5326\end{array}$ & $\begin{array}{l}\text { PEG } \\
N=25,388\end{array}$ \\
\hline Sex, female & 95.2 & 95.5 & 90.6 & 91.4 & 88.5 & 88.1 \\
\hline Age, $18-54$ years & 71.5 & 72.8 & 45.7 & 46.8 & 34.9 & 32.9 \\
\hline At least one cardiovascular risk factor ${ }^{\mathrm{a}}$ & 60.5 & 59.1 & 55.5 & 51.8 & 76.7 & 79.7 \\
\hline History of hospitalization for CVD & 5.4 & 4.3 & 6.5 & 6.4 & 11.6 & 14.6 \\
\hline Any revascularization procedures & 1.5 & 1.3 & 3.2 & 3.4 & 6.4 & 8.1 \\
\hline Aspirin and other antiplatelets & 14.6 & 12.1 & 19.9 & 19.7 & 11.5 & 13.8 \\
\hline Statins & 17.3 & 15.7 & 23.9 & 22.7 & 21.3 & 22.6 \\
\hline Antihypertensives & 42.1 & 36.1 & 49.6 & 46.5 & 56.1 & 61.9 \\
\hline Antidiabetics & 7.0 & 6.0 & 9.6 & 9.8 & 9.9 & 12.6 \\
\hline Anticoagulants & 3.2 & 2.8 & 16.4 & 15.5 & 20.3 & 28.0 \\
\hline Hyperlipidemia diagnosis & 8.8 & 7.7 & 7.1 & 6.8 & 43.8 & 44.3 \\
\hline Hypertension diagnosis & 13.7 & 13.7 & 21.0 & 21.8 & 54.7 & 60.2 \\
\hline Obesity $^{\mathrm{b}}$ & 17.4 & 21.2 & 0.8 & 0.6 & NA & NA \\
\hline Obesity treatments & 6.6 & 5.5 & 1.2 & 1.3 & 0.0 & 0.1 \\
\hline Diabetes diagnosis & 6.7 & 5.4 & 8.2 & 8.7 & 17.9 & 21.8 \\
\hline Chronic renal disease diagnosis & 3.6 & 3.3 & 1.1 & 1.3 & 10.9 & 14.9 \\
\hline Cancer & 5.9 & 6.6 & 9.1 & 10.5 & 27.3 & 40.9 \\
\hline \multicolumn{7}{|l|}{ Smoking $(\mathrm{CPRD} / \mathrm{THIN} \text { only })^{\mathrm{c}}$} \\
\hline Never smoker & 49.1 & 48.2 & NA & NA & NA & NA \\
\hline Former smoker & 31.0 & 29.5 & NA & NA & NA & NA \\
\hline Current smoker & 19.2 & 21.2 & NA & NA & NA & NA \\
\hline Unknown & 0.7 & 1.1 & NA & NA & NA & NA \\
\hline COPD diagnosis & 3.6 & 3.3 & 3.7 & 4.3 & 35.2 & 36.6 \\
\hline Asthma without COPD diagnosis & 17.9 & 13.8 & 8.1 & 6.4 & 5.5 & 4.9 \\
\hline Recent hospitalization $^{\mathrm{d}}$ & 6.4 & 7.6 & 2.7 & 2.5 & 5.9 & 15.9 \\
\hline $\begin{array}{l}\text { Number of outpatient medical visits with constipation diag- } \\
\text { noses (CPRD/THIN/SNR/GePaRD only) } \geq 1^{\mathrm{e}}\end{array}$ & 76.9 & 40.4 & 34.0 & 7.4 & 48.1 & 13.9 \\
\hline $\begin{array}{l}\text { Number of medical visits with IBS diagnoses (CPRD/THIN/ } \\
\text { SNR/GePaRD only) } \geq 1^{\text {e }}\end{array}$ & 34.5 & 17.8 & 11.8 & 2.3 & 19.9 & 5.1 \\
\hline $\begin{array}{l}\text { Number of unique other gastrointestinal-related outpatient } \\
\text { diagnoses (CPRD/THIN/SNR/GePaRD only), } 1-12^{\mathrm{f}}\end{array}$ & 44.3 & 31.1 & 46.9 & 32.6 & 83.0 & 69.0 \\
\hline $\begin{array}{l}\text { Constipation inpatient diagnosis (CPRD/ISD/SNR/GePaRD } \\
\text { only) }\end{array}$ & 38.2 & 5.5 & 7.6 & 1.9 & 19.5 & 12.8 \\
\hline IBS inpatient diagnosis (CPRD/ISD/SNR/GePaRD only) & 5.5 & 1.7 & 1.8 & 0.4 & 2.1 & 0.5 \\
\hline $\begin{array}{l}\text { Other gastrointestinal-related inpatient diagnosis (CPRD/ } \\
\text { ISD/SNR/GePaRD only) }\end{array}$ & 39.0 & 20.4 & 24.3 & 14.6 & 39.8 & 37.6 \\
\hline Prescription or dispensing for opioid medications ${ }^{\mathrm{g}}$ & 36.5 & 36.2 & 25.3 & 31.3 & 20.8 & 44.0 \\
\hline Chronic opioid use $^{\mathrm{h}}$ & 33.8 & 30.2 & 21.9 & 23.3 & 18.1 & 36.7 \\
\hline \multicolumn{7}{|l|}{ Economic deprivation category (UK only) } \\
\hline Q1 (least deprived) & 22.1 & 19.3 & NA & NA & NA & NA \\
\hline Q2 & 19.0 & 18.4 & NA & NA & NA & NA \\
\hline Q3 & 20.1 & 20.5 & NA & NA & NA & NA \\
\hline Q4 & 19.0 & 20.9 & NA & NA & NA & NA \\
\hline Q5 (most deprived) & 18.8 & 19.7 & NA & NA & NA & NA \\
\hline Unknown & 1.1 & 1.1 & NA & NA & NA & NA \\
\hline \multicolumn{7}{|l|}{ Economic deprivation category (SNR only) } \\
\hline Q1 (least deprived) & NA & NA & 24.9 & 21.8 & NA & NA \\
\hline Q2 & NA & NA & 24.6 & 24.7 & NA & NA \\
\hline
\end{tabular}


Table 1 (continued)

\begin{tabular}{|c|c|c|c|c|c|c|}
\hline \multirow[t]{2}{*}{ Covariate } & \multicolumn{2}{|c|}{ UK (CPRD, THIN, ISD) } & \multicolumn{2}{|l|}{ SNR } & \multicolumn{2}{|l|}{ GePaRD } \\
\hline & $\begin{array}{l}\text { Prucalopride } \\
N=2521\end{array}$ & $\begin{array}{l}\text { PEG } \\
N=12,603\end{array}$ & $\begin{array}{l}\text { Prucalopride } \\
N=3194\end{array}$ & $\begin{array}{l}\text { PEG } \\
N=16,769\end{array}$ & $\begin{array}{l}\text { Prucalopride } \\
N=5326\end{array}$ & $\begin{array}{l}\text { PEG } \\
N=25,388\end{array}$ \\
\hline Q3 & NA & NA & 25.3 & 24.5 & NA & NA \\
\hline Q4 (most deprived) & NA & NA & 25.3 & 29.0 & NA & NA \\
\hline
\end{tabular}

Data are presented as \%

$B M I$ body mass index, $C O P D$ chronic obstructive pulmonary disease, $C P R D$ Clinical Practice Research Datalink, $C V D$ cardiovascular disease, GePaRD German Pharmacoepidemiological Research Database, IBS irritable bowel syndrome, ISD Information Services Division, MACE major adverse cardiovascular events, NA not applicable, PEG polyethylene glycol 3350, SNR Swedish National Registers, THIN The Health Improvement Network

${ }^{a}$ History of CVD (including all MACE component endpoints), hypertension, smoking, hyperlipidemia, diabetes, aged $>55$ years, or $\mathrm{BMI}>30 \mathrm{~kg} / \mathrm{m}^{2}$

${ }^{b}$ Not available for ISD; for CPRD and THIN defined as whether or not BMI $>30 \mathrm{~kg} / \mathrm{m}^{2}$ documented within the prior 3 years of cohort entry and for SNR based on recorded diagnosis of obesity prior to cohort entry date

${ }^{c}$ Identified using the information reported closest in time to the cohort entry date within the prior 10 years

${ }^{\mathrm{d}}$ Any hospitalization, regardless of diagnosis, in the 14 days immediately preceding cohort entry

${ }^{e}$ Diagnoses occurring any time before taking the index study medication

${ }^{\mathrm{f}}$ Total number of unique other gastrointestinal diagnoses occurring any time before the index study medication. Other gastrointestinal diagnoses considered were esophageal conditions, gastroduodenal conditions, appendicitis, hernias, intestinal conditions, peritonitis, liver conditions, biliary conditions, pancreatic conditions, gastrointestinal hemorrhage, malabsorption, and inflammatory bowel disease

${ }^{g}$ Occurring in the 6 months before cohort entry

${ }^{\mathrm{h}}$ Chronic opioid use was defined as more than one unique prescription or dispensing (i.e. occurring on separate days) for an opioid during the 12 months before the index date

prescribing and follow-up assessments were part of routine clinical care and not influenced by the study).

The prucalopride cohort included patients with a dispensing/prescription for prucalopride, at least 12 months of data available in the data source prior to the first dispensing/ prescription, and no evidence of prior use of prucalopride (see Fig. S-1 in ESM 2). The PEG cohort included patients with a dispensing/prescription for PEG, at least 12 months of data available in the data source prior to the first dispensing/ prescription, and no evidence of prior use of PEG (see Fig. $\mathrm{S}-2$ ). Individuals aged $<18$ years at the time of first prucalopride or PEG dispensing/prescription or whose first prucalopride or PEG dispensing/prescription was for $\leq 4$ days were excluded, as these patients may have been receiving treatment for acute constipation or procedural preparation. Individuals in the PEG cohort who switched to prucalopride were censored from the PEG cohort and entered the prucalopride cohort if all other inclusion/exclusion criteria were met. Individuals in the prucalopride cohort who switched to PEG were censored from the prucalopride cohort and, if all inclusion/exclusion criteria were met, were eligible to be selected for the PEG cohort. Patients could switch only once, as they would not be new users thereafter.

The first dispensing/prescription of prucalopride or PEG was the index prescription, and the date dispensed or prescribed was the index date.

\subsubsection{Removal of Duplicate Patients in the UK Databases}

Approximately $10-15 \%$ of CPRD and THIN practices are located in Scotland and, consequently, had to be excluded from the CPRD and THIN cohorts since they were also included in the ISD Scotland database. Also, a percentage of practices are known to be included in both the CPRD and THIN. The approach taken to ensure removal of duplicate patients involved (1) eliminating all the Scottish practices from the CPRD and THIN and (2) identifying the remaining potential duplicated practices by identifying patients thought to be the same individual in the CPRD and THIN and then excluding all patients belonging to the same practice (in CPRD or THIN) as the identified duplicate patients. We used a modified six-step algorithm based on the one published by Cai et al. [9]. In case of duplication, CPRD practices were retained and their equivalents in THIN were removed.

\subsubsection{Matching PEG Initiators to Prucalopride Initiators}

Up to five different PEG initiators were matched to each prucalopride initiator. Once a PEG initiator was matched to one prucalopride initiator, he or she was not eligible to be matched to any other prucalopride initiators. The match was performed by sex, calendar year of the index date, and year of birth ( \pm up to 10 years, if needed). 
In Sweden, it was found that PEG was prescribed to patients after surgery (drug prescribed by surgeon) much more frequently than was prucalopride. Thus, patients from the SNR were additionally matched on recent hospitalization and the type of prescriber physician to increase the likelihood of capturing patients with chronic constipation rather than constipation prophylaxis related to surgery.

All prucalopride patients with at least one matched PEG comparator were retained for the analysis.

\subsection{Variables}

Exposure to prucalopride or PEG was ascertained from general practitioner (GP) prescriptions in the CPRD and THIN data, and from outpatient dispensing in the ISD, SNR, and GePaRD data. Cardiovascular risk factors and other covariates were identified from healthcare utilization codes recorded before the index date. Codes were harmonized across data sources.

\subsubsection{Study Exposures: Treatment Episodes of Prucalopride or PEG}

Drug use was identified using the conventional drug coding schemes at each data source, assuming patient adherence aligned with the prescribing or dispensing patterns. A treatment episode was defined as consecutive dispensings/prescriptions of the index medication (i.e. the medication dispensed or prescribed on the index date), including a 7-day extension period after the drug was discontinued. Overlapping time of two prescriptions of the same drug was counted only once in the three data sources from the UK, while stockpiling was used in Sweden and Germany. Gaps between prescriptions or dispensing lasting more than 7 days ended the treatment episode. All prucalopride use within the study period was accounted for as exposed time, including the gaps $<7$ days (i.e. regardless of the duration of the prescription/dispensing). On the other hand, short episodes of PEG (lasting < 12 days) during follow-up were considered proxies of indications other than chronic constipation (e.g. acute constipation or preparation for a colonoscopy) and were ignored, as the comparison cohort was intended to have chronic constipation and PEG is assumed to be neutral in terms of risk of MACE (see Fig. S-3 in ESM 2).

\subsubsection{Follow-Up}

Accrual of time at risk of current use for a study medication was terminated if there was a prescription or dispensing for the other study medication. Time at risk did not accrue beyond 7 days after the end of the last prescription/dispensing for the index medication, date of death, date of first occurrence of the endpoint under evaluation, termination of enrolment in the health plan or system, or end of study period.

For the primary analysis, time at risk from current use for prucalopride initiators and PEG initiators started on the day of index prescription or dispensing and was accumulated across all episodes of the index medication (e.g. time at risk from current use began again if a patient re-started a drug after a gap in use of $>7$ days) and ended at a termination date, as detailed above.

\subsubsection{Outcomes}

The main composite cardiovascular endpoint of interest for this study was based on the classical MACE endpoint definition used in randomized controlled trials. This composite cardiovascular endpoint included hospitalization for nonfatal acute myocardial infarction, hospitalization for nonfatal stroke, and in-hospital cardiovascular death.

As we anticipated a lack of information on cause of outof-hospital death and on outpatient diagnoses in some of the selected data sources, the MACE endpoint for the main analyses included in-hospital but not out-of-hospital cardiovascular deaths. This approach followed the FDA recommendations for consistent classification (i.e. the ascertainment of homogeneous events across all selected data sources is preferable to defining endpoints based on the maximum available information in each data source).

In all data sources, potential study endpoints were identified by electronic algorithms combining harmonized operational definitions and lists of diagnostic and procedural codes (using International Classification of Diseases codes and/or local dictionaries). Using these definitions, each data source was searched for evidence indicating the occurrence of an endpoint of interest. Validation of study endpoints was conducted in the UK data sources and is reported elsewhere (Cainzos-Achirica et al. [10]).

\subsection{Statistical Analyses}

Figure 1 depicts the main features of the study analyses and the critical path for their implementation. Patient-level data were held at each research center. Database-specific analyses implemented by the research centers were conducted using SAS version 9.3 or higher (SAS Institute, Inc., Cary, NC, USA) for the CPRD, SNR, ISD Scotland, and GePaRD; Stata 12 (StataCorp LP, College Station, TX, USA) was used for THIN data. From the matched cohorts, the PS was estimated at the index date for each initiator of prucalopride or PEG using relevant covariates. Then, PS distribution was used to trim both cohorts to obtain more comparable cohorts. Finally, the deciles of the PS were derived, and balance of the potential confounding factors between cohorts was checked. Stratification on the deciles of the PS was used 


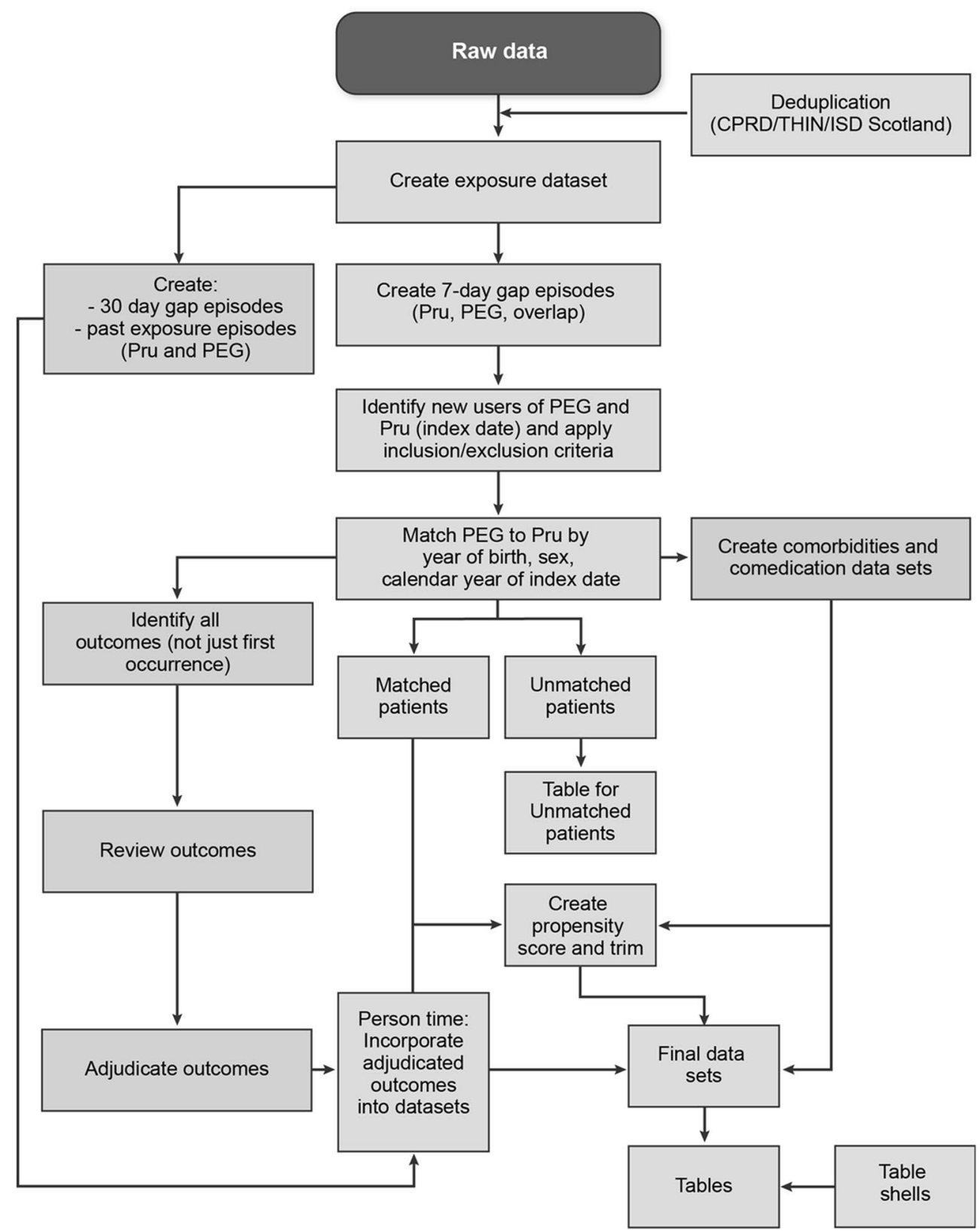

CPRD = Clinical Practice Research Datalink; ISD = Information Services Division; PEG = polyethylene glycol 3350; Pru = prucalopride; THIN = The Health Improvement Network.

Fig. 1 Study design and implementation of analyses

to obtain adjusted estimates for the main study [5]. For each data source, the following characteristics of all variables of interest were described for each cohort after trimming: for categorical variables, the frequency (i.e. count and proportion, by number and by person-years) with the characteristic of interest; for continuous variables, the mean and standard deviation, minimum, maximum, median, and quartiles.

\subsubsection{Modelling the Propensity to Receive Prucalopride}

PS indicating likelihood of receiving versus not receiving prucalopride were estimated using logistic regression, modelling prucalopride initiation versus PEG initiation as the outcome and including matching variables and potential confounders-age, sex, calendar year of the index date, comorbidities, and indicators for cardiovascular risk and gastrointestinal morbidities—as the independent variables. 
All variables related to the outcome were considered for the estimation of the PS, regardless of whether they were related to the exposure, as stated by Brookhart et al. [11]. Therefore, variables in an outcome model (MACE) with an odds ratio $(\mathrm{OR})>1.25$ or $<0.8$ were selected to estimate the PS for each patient, separately in each data source. The matching variables (i.e. age, sex, and calendar year) were kept in the PS, as was any variable considered clinically important by each research partner. Even though the matching variables were balanced, they were included as they could have become unbalanced upon controlling for other factors in the PS.

For summary variables and their individual components, if the model converged, only the individual components of the summary variable were included. If the model with individual components did not converge, only the summary variable was included. For highly correlated variables (e.g. a diagnosis and its treatments or in-hospital and out-of-hospital diagnoses of a condition), if the model converged, the correlated variables were included. If the model with the correlated variables did not converge, a variable combining the information (e.g. condition untreated, condition treated with drug A, condition treated with drug B) was created, information was collapsed (e.g. collapsed in- and out-ofhospital data), or, if needed, one of the correlated variables was removed from the model. Variables too strongly associated with the exposure were excluded if the OR in the PS model was $>10$ or $<0.1$ to avoid excessive separation of both populations, which would have resulted in excessive trimming and loss of prucalopride patients in the analysis. Consequently, prior use of a study drug (i.e. prucalopride or PEG) was not included in the PS due to high correlation with the exposure of interest. See Table S-1 in ESM 3 for variables included in the PS for each data source.

From the fitted PS model, a PS was calculated for each patient. In subjects who switched between study drugs, the PS was recalculated at the time of its new index dispensing/ prescription, using the corresponding new values for covariates at the new index date.

2.5.1.1 Trimming Trimming of the population based on extreme values of the PS was conducted to obtain two comparable populations by excluding patients who had extreme PS relative to the other group (i.e. were unlikely to have been prescribed one or the other study drug). The 1st percentile of the PS distribution was identified among those exposed to prucalopride, and all prucalopride or PEG initiators whose PS was below this value were excluded. Similarly, the 99th percentile of the PS distribution among the PEG cohort was identified, and all patients in both cohorts whose PS was above this value were excluded.
2.5.1.2 Calculation of PS Deciles The cut-off points of the deciles of the PS distribution in the prucalopride cohort were identified and used to categorize patients in both study cohorts.

2.5.1.3 Balance of the Propensity Score The balance of the PS was assessed within each PS decile using the standardized mean difference (SMD) [12], which is based on the distribution of each variable by cohort. Published literature on PS recommends that variables are considered balanced if they have an SMD $<0.2$ [12].

When balance was not achieved in variables clinically considered to be potential confounders, the following approach was employed: (1) for variables already included in the PS model, an ad hoc solution was sought to reduce imbalance (e.g. increase trimming, include interaction terms); (2) for variables not included in the PS, the variable was forced into the PS model, and balance was reassessed. After refining the PS to improve balance, the PS model was considered valid if the averaged SMD across PS deciles for variables included in the PS was $<0.2$.

2.5.1.4 Missing Values The frequency of missing information is shown in the descriptive analyses tables in a separate category.

\subsection{Study Size}

A cohort size of 10,950 prucalopride initiators was projected to be sufficient to provide $80 \%$ power to reject the hypothesis of an upper limit of the risk ratio $>3.0$, assuming the true risk ratio to be 1.0 and an expected background incidence for the primary combined cardiovascular endpoint (MACE) of 2 per 1000 person-years. This upper limit margin of safety was agreed between the sponsor and the FDA. The number of prucalopride initiators was based on an average currentuse follow-up time for prucalopride initiators of 130 days. Calculations were made using the formulas in Episheet [13].

\section{Results}

Results describing the study population characteristics and balance of covariates in the PEG and prucalopride cohorts are presented in this manuscript. Results for the primary pooled analysis of MACE and all study sensitivity analyses are presented separately (see Gilsenan et al. [5]).

\subsection{Study Population and Cohort Attrition}

A total of 2,512,085 patients were identified from all five data sources (15,492 prucalopride, 2,496,593 PEG). After exclusion criteria were applied and up to five PEG patients 
were matched to each prucalopride patient, the overall study population included patients with chronic constipation treated with prucalopride $(n=12,030)$ or PEG $(n=59,985)$. Figure 2 summarizes the attrition process for Germany, Sweden, and the UK.

In total, 60 prucalopride initiators in $\mathrm{GePaRD}$ were excluded from the analyses because no suitable PEG initiators were identified for matching. No prucalopride initiators were excluded in the UK or SNR due to lack of suitable PEG initiators for matching. Overall, 989 prucalopride initiators (8.2\%) and 5225 PEG initiators (8.7\%) were excluded due to trimming, representing those patients who had an extreme PS relative to the other group, and hence improving the comparability of the study cohorts.

Overall, 5715 initiators of prucalopride and 29,372 PEG initiators were included in the study.

\subsection{Propensity Score Creation and Cohort Trimming}

In the CPRD, all variables were initially included in the PS model. However, inpatient and outpatient constipation diagnoses showed a high correlation with the exposure and were therefore excluded from the final PS model for CPRD. Trimming of the population based on the PS improved the balance of the included variables (i.e. the SMD decreased). The model achieved good balance for the included variables.

In THIN, all covariates associated with MACE were initially included in the PS model. Due to the strong association of "number of outpatient constipation diagnosis" with the exposure, with an OR $>10$ in the PS model, this variable was finally excluded from the model, which reduced exclusions due to trimming. The model achieved good balance for the included variables.
In ISD Scotland (where outpatient diagnoses were not available), the trimming of the PS including all a prioriselected variables improved the balance for "irritable bowel syndrome [IBS] inpatient diagnosis" and "other gastrointestinal-related inpatient diagnosis," allowing these to be retained in the model. Constipation inpatient diagnosis was dropped, as it was too strongly predictive of exposure. The model achieved good balance for the included variables.

In SNR, an alternative matching model was built to increase the balance that included type of prescriber and recent hospitalization. The PS model did not include the variable outpatient medical visits with a constipation diagnosis, as it had an OR $>10$. The model achieved good balance for the included variables.

In GePaRD, four different approaches to estimate the PS were used:

1. Only variables in an outcome model for MACE with an OR $>1.25$ or $<0.8$ were selected to estimate the PS (same approach as UK and SNR)

2. All variables were selected to estimate the PS

3. All variables were selected to estimate the PS, but inpatient and outpatient diagnoses of constipation, IBS, and other gastrointestinal-related diagnoses were collapsed

4. Only variables that were thought to be associated with the outcome MACE were selected to estimate the PS (excluding markers of potential indications such as constipation, IBS, other gastrointestinal-related diagnosis, colonoscopy, and other drugs for constipation).

Ultimately, method 4 was selected because it provided the most comparable cohorts. ESM 3 presents the PS distributions for each data source.
Fig. 2 Cohort attrition. GePaRD German Pharmacoepidemiological Research Database, $P E G$ polyethylene glycol 3350, SNR Swedish National Registers

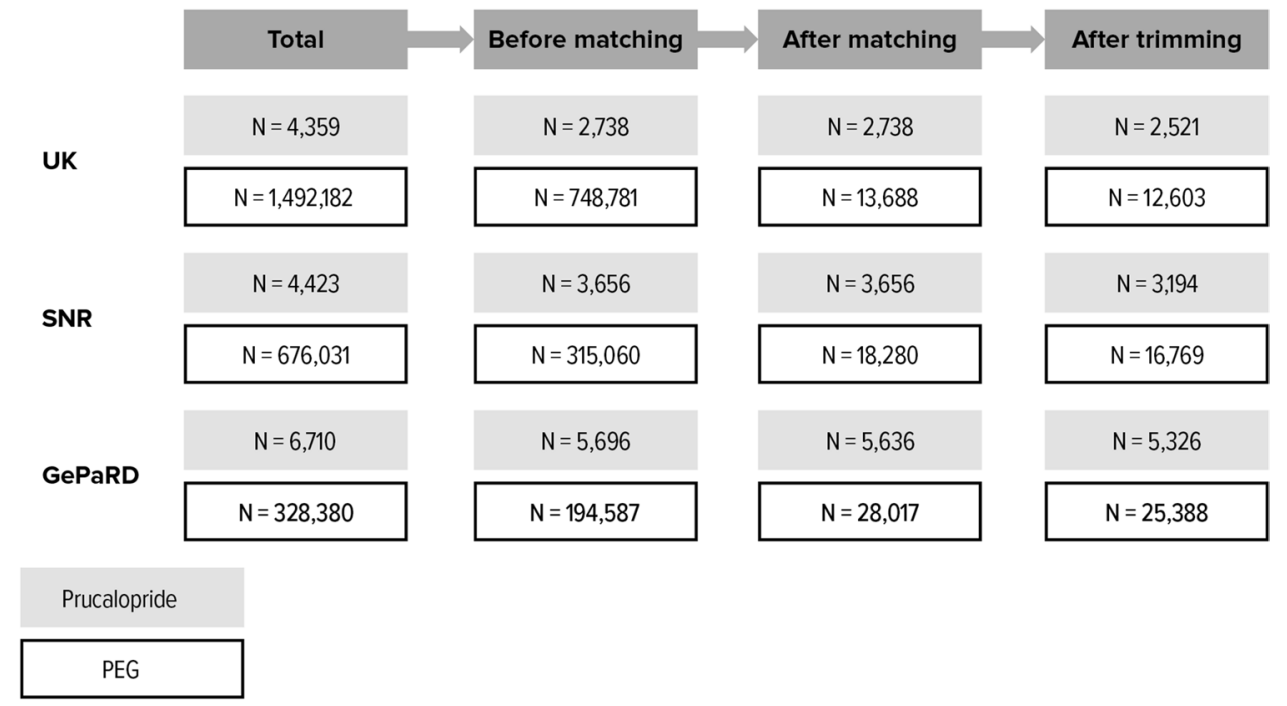




\subsection{Effect of Trimming}

The study population after the trimming process included 11,041 patients treated with prucalopride and 54,760 patients treated with PEG. Of these, 5120 matched patients were included from the CPRD, 3044 from THIN, 6960 from the ISD, 19,963 from the SNR, and 30,714 from GePaRD.

\subsection{Balance of Study Covariates}

In the UK and Sweden, most covariates achieved good balance (SMD < 0.2) after trimming and PS decile stratification. However, the following variables did not achieve good balance: number of outpatient medical visits with a constipation diagnosis, number of constipation inpatient diagnoses, number of outpatient medical visits with an IBS diagnosis, number of IBS inpatient diagnoses, number of unique other gastrointestinal-related outpatient diagnoses, and number of other gastrointestinal-related inpatient diagnoses. As described above, some of these variables were not included in the PS models due to high correlation with exposure to the study drugs; however, their elimination from the PS model should not create confounding as they are not considered predictors of major cardiovascular outcomes.

\subsection{Distribution of Demographic, Cardiovascular, and Gastrointestinal Covariates in Germany, Sweden, and the UK}

Table 1 describes the prevalence of demographics, cardiovascular risk factors, and gastrointestinal-related conditions of interest in both cohorts, separately for Germany, Sweden, and the UK, after matching and trimming.

In the UK, $28.5 \%$ of prucalopride initiators and $27.2 \%$ of PEG initiators were aged $\geq 55$ years. In the GePaRD, patients were older, the corresponding proportions being $65.1 \%$ and $67.1 \%$. In the SNR, patients were also older than in the UK: $54.3 \%$ of prucalopride initiators and $53.2 \%$ of PEG initiators were aged $\geq 55$ years. Most study participants were women, ranging from $88.1 \%(\mathrm{GePaRD})$ to $95.5 \%$ (UK).

In the UK, $5.4 \%$ of those exposed to prucalopride and 4.3\% of those exposed to PEG had a prior history of hospitalization for cardiovascular diseases. The corresponding figures for SNR were 6.5\% and 6.4\%. In contrast, the proportions for GePaRD were $11.6 \%$ for prucalopride initiators and $14.6 \%$ for PEG initiators. Similarly, in the UK, 5.9\% of those in the prucalopride cohort and $6.6 \%$ of those in the PEG cohort had a prior history of any cancer. In SNR, the figures were $9.1 \%$ and $10.5 \%$. On the other hand, the prevalence of cancer in GePaRD was much higher: $27.3 \%$ in the prucalopride cohort and $40.9 \%$ in the PEG cohort.

\subsection{Data Sources to Be Included for Pooled Analyses}

In view of this disparate clinical profile, it was not considered appropriate to combine estimates from GePaRD with those from the other data sources in the pooled study analyses for MACE.

\section{Discussion}

The study was designed as a multi-database study in which the primary results would be pooled across the participating data sources. It was originally intended to include GePaRD data from Germany; however, a disparate clinical profile of German patients compared with Sweden and the UK, particularly in initiators of PEG, was observed, likely due to the reimbursement policies in Germany (see ESM 4). This resulted in an imbalance between initiators of prucalopride and PEG in Germany, particularly for variables such as history of cancer and recent hospitalization, and, more generally, a different patient profile between Germany and the UK and Sweden.

This study is an example of performance of PS in a real-life setting with considerable confounding. GePaRD investigators attempted several approaches to develop a PS to achieve comparability between the two study cohorts. High-dimensional PS techniques were conducted to adjust for additional variables, and various degrees of trimming of the cohorts were done to improve comparability of patients. However, investigators were unable to overcome the substantial baseline differences in risk of MACE observed between study cohorts, and the decision was made not to pool GePaRD data with those of the UK and Swedish data sources.

Also, of note, the unmatched population of Swedish PEG initiators was similar to that for GePaRD in age and comorbidities. However, local clinical input and review of the data revealed that many of the unmatched PEG initiators were not being treated for chronic constipation but receiving a prescription for PEG to prevent postsurgical constipation. The inclusion of two additional matching variables (i.e. recent hospitalization and type of prescribing physician specialty) enabled identification of PEG initiators that was more comparable to the group of Swedish initiators of prucalopride and that was suitable for use in the matched analyses in SNR.

In general, for the UK and SNR, balance was achieved among study covariates, except for those that were gastrointestinal related. Imbalances for gastrointestinal-related variables, which are unlikely to be strong risk factors for MACE, were not unexpected because prucalopride is reserved for patients with chronic constipation that is insufficiently treated with other laxatives.

In the CPRD and THIN data sources from the UK, where prescriptions are captured through GP prescribing, any 
prescription by a specialist would not be captured in the corresponding database. Nevertheless, specialists in England commonly write first prescriptions, while subsequent prescriptions are usually transferred to the GP. Because most patients with chronic constipation would be expected to be prescribed prucalopride/PEG more than once, most exposures to the study drugs would be expected to have been captured by the data sources, although perhaps misclassified as new use. In Scotland, clinical specialists routinely implement changes to a patient's prescribing for chronic conditions by asking the patients' GP to do the prescribing, so there is unlikely to be any uncaptured outpatient prescribing for prucalopride. The prescribing database used in Scotland was a record of dispensed prescriptions, which is a better record of exposure than prescribed prescriptions [14]. Nevertheless, as is the case in any study using prescribing or dispensing as a measure of exposure, actual ingestion of medications cannot be measured and may be misclassified.

Efforts were made to harmonize the definitions of exposure periods and key study variables. Still, nuances were allowed for endpoint definitions, to adapt the core definitions to the local coding practices and thus avoid outcome misclassification, and for some covariates; these were minor, and no relevant impact on the study results was anticipated.

Information on some potential confounders (e.g. smoking, obesity) was available in some but not all data sources; where available, the cohorts were well balanced on these variables. Information on other potential confounders, such as use of over-the-counter aspirin or anti-inflammatory drugs, was not captured in these data sources. In addition, as with all studies relying on existing records, recording errors that over- or under-state a variable are possible; we consider it unlikely that these errors would be differentially distributed between the prucalopride and PEG cohorts.

\section{Conclusions}

The overarching aim of this study was to estimate the risk of MACE among initiators of prucalopride and to compare it with initiators of PEG. As patient characteristics influence the choice of drug, it is important to reduce confounding by indication by making both exposure groups comparable. Data from four data sources in Europe (three in the UK and one in Sweden) were used in the pooled analyses; data from Germany could not be used due to substantial differences in prescribing practices that resulted in populations that were too different to be pooled. Modelling propensity to receive prucalopride yielded a good balance of main potential confounders for MACE but was unable to achieve good balance for gastrointestinal-related variables, which are unlikely to be strong risk factors for MACE.
Acknowledgements The authors thank Antonio Gonzalez (CEIFE), who contributed to the statistical analyses of THIN data and report preparation, and Jennifer Bartsch (RTI-HS), who contributed to the statistical programming for the CPRD data. The authors are also grateful for input on the design from Patricia Tennis, Kenneth Rothman, and Eileen Ming. Helle Kieler contributed to the study as a primary investigator in Sweden. The authors thank Abenah Harding from RTI-HS and Leah McGrath, formerly from RTI-HS, for their project management support. Kate Lothman of RTI Health Solutions provided medical writing services, which were funded by Shire Pharmaceuticals, now part of the Takeda group of companies. This study is based in part on data from the CPRD obtained under license from the UK Medicines and Healthcare products Regulatory Agency. The data are provided by patients and collected by the National Health Service as part of their care and support. The interpretation and conclusions contained in this study are those of the authors alone. We thank the statutory health insurance providers in Germany, AOK Bremen/Bremerhaven, DAK-Gesundheit, and Die Techniker (TK), for contributing the data for this analysis. We acknowledge the Electronic Data Research and Innovation Service (eDRIS) of ISD Scotland for providing the ISD data. We also acknowledge Quintiles IMS (now IQVIA) for providing access to THIN data.

\section{Compliance with Ethical Standards}

Conflict of interest This study was performed under a research contract between RTI Health Solutions and Shire, now part of the Takeda group of companies, and was funded by Shire. The research contract granted independent publication rights to the research team, and the content of the publications was developed independently from the study sponsor. Alicia Gilsenan, Joan Fortuny, Estel Plana, Ryan Ziemiecki, and Elizabeth B. Andrews are salaried employees of RTI Health Solutions. Miguel Cainzos-Achirica was a salaried employee of RTI Health Solutions when this research was conducted and is currently affiliated with the Hospital Universitari de Bellvitge Department of Cardiology, Feixa Llarga, Hospitalet de Llobregat, Barcelona, Spain. Luis Garcia-Rodriguez, Oscar F. Cantero, and Ana Ruigómez are employed at the Spanish Centre for Pharmacoepidemiologic Research (CEIFE Madrid, Spain). Robert W. V. Flynn and Professor Thomas M. MacDonald are employees of the University of Dundee, an academic organization that received financial support under the research contract between RTI Health Solutions and Shire, now part of the Takeda group of companies, to carry out the study. Robert W. V. Flynn has received research grants from Novartis and GlaxoSmithKline. Professor Thomas M. MacDonald is the director of MEMO Research at the University of Dundee, an organization that has received research funding from Pfizer, Novartis, Amgen, Ipsen, GlaxoSmithKline, Teijin, and Menarini, mostly for post-licensing studies requested by regulatory authorities, and has personally received honoraria for educational lectures from Takeda. He has been the principal investigator on trials paid for by Pfizer, Novartis, Ipsen, Teijin, GlaxoSmithKline, and Menarini (mostly post-licensing regulatory requested studies). In the last 3 years, he has received consulting fees from Novartis. Pär Karlsson and Love Linnér are employed at the Centre for Pharmacoepidemiology, Karolinska Institutet, which receives grants from several entities (pharmaceutical companies, regulatory authorities and contract research organizations) for performance of drug safety and drug utilization studies. These entities had no role in the data collection and analysis and were not involved in the interpretation of results, writing, revision, and approval of the manuscript. Miguel Cainzos-Achirica has participated in a research study funded by a non-conditioned research grant from Vifor. Tania Schink and Bianca Kollhorst are working at an independent, non-profit research institute, the Leibniz Institute for Prevention Research and Epidemiology - BIPS. Unrelated to this study, BIPS occasionally conducts studies financed by the pharmaceutical 
industry. Almost exclusively, these are post-authorization safety studies requested by health authorities. The design and conduct of these studies as well as the interpretation and publication are not influenced by the pharmaceutical industry.

Funding This study was funded by Shire, now part of the Takeda group of companies.

Ethical approval All relevant authorities reviewed and approved the study on ethical grounds in the UK, Germany, and Sweden.

Research involving human participants Not applicable; the data used in this study were obtained from databases of medical records and not directly from human subjects.

Open Access This article is distributed under the terms of the Creative Commons Attribution-NonCommercial 4.0 International License (http://creativecommons.org/licenses/by-nc/4.0/), which permits any noncommercial use, distribution, and reproduction in any medium, provided you give appropriate credit to the original author(s) and the source, provide a link to the Creative Commons license, and indicate if changes were made.

\section{References}

1. Dennison C, Prasad M, Lloyd A, Bhattacharyya SK, Dhawan R, Coyne K. The health-related quality of life and economic burden of constipation. Pharmacoeconomics. 2005;23(5):461-76.

2. Wald A, Scarpignato C, Kamm MA, Mueller-Lissner S, Helfrich I, Schuijt C, et al. The burden of constipation on quality of life: results of a multinational survey. Aliment Pharmacol Ther. 2007;26(2):227-36.

3. Gershon MD, Tack J. The serotonin signaling system: from basic understanding to drug development for functional GI disorders. Gastroenterology. 2007;132(1):397-414.

4. FDA. FDA briefing document, New Drug Application 210166 for prucalopride: Gastrointestinal Drug Advisory Committee meeting. 18 October 2018. https://www.fda.gov/downloads/Advis oryCommittees/CommitteesMeetingMaterials/Drugs/Gastrointe
stinalDrugsAdvisoryCommittee/UCM623493.pdf. Accessed 26 Oct 2018.

5. Gilsenan A, Fortuny J, Cainzos-Achirica M, Cantero AF, Flynn RWV, Garcia-Rodriguez L, et al. Cardiovascular safety of prucalopride in patients with chronic constipation: a multinational population-based cohort study. Drug Saf. 2019. https://doi. org/10.1007/s40264-019-00835-0.

6. ENCePP. Cohort study of the relative incidence of major cardiovascular events among patients initiating prucalopride versus a matched comparator cohort (EUPAS9200). European Network of Centres for Pharmacoepidemiology and Pharmacovigilance; July 2018. http:// www.encepp.eu/encepp/viewResource.htm?id=22643. Accessed 18 Dec 2018.

7. Ray WA. Evaluating medication effects outside of clinical trials: new-user designs. Am J Epidemiol. 2003;158(9):915-20.

8. Shire Pharmaceuticals. Annex I. Summary of product characteristics. Resolor (prucalopride). 2009. http://www.ema.europa.eu/ docs/en_GB/document_library/EPAR_-_Product_Information/ human/001012/WC500053998.pdf. Accessed 19 Dec 2018.

9. Cai B, Xu W, Bortnichak E, Watson DJ. An algorithm to identify medical practices common to both the General Practice Research Database and The Health Improvement Network database. Pharmacoepidemiol Drug Saf. 2012;21(7):770-4.

10. Cainzos-Achirica M, Ruigómez A, MacDonald TM, GarcíaRodríguez L, Fortuny J, Flynn RWV, et al. Validation of major cardiovascular events in a multi-database post-authorization safety study of prucalopride. 35th International Conference on Pharmacoepidemiology \& Therapeutic Risk Management, August 24-28 2019.

11. Brookhart MA, Schneeweiss S, Rothman KJ, Glynn RJ, Avorn J, Sturmer T. Variable selection for propensity score models. Am J Epidemiol. 2006;163(12):1149-56.

12. Austin PC. Assessing balance in measured baseline covariates when using many-to-one matching on the propensity-score. Pharmacoepidemiol Drug Saf. 2008;17(12):1218-25.

13. Rothman KJ. Episheet—spreadsheets for the analysis of epidemiologic data. October 4, 2012. http://krothman.hostbyet2.com/ Episheet.xls. Accessed 19 Dec 2018.

14. Beardon PH, McGilchrist MM, McKendrick AD, McDevitt DG, MacDonald TM. Primary non-compliance with prescribed medication in primary care. BMJ. 1993;307(6908):846-8.

\section{Affiliations}

\section{Joan Fortuny ${ }^{1} \cdot$ Alicia Gilsenan $^{2} \cdot$ Miguel Cainzos-Achirica $^{1,7}$. Oscar F. Cantero ${ }^{3}$ - Robert W. V. Flynn ${ }^{4}$. Luis Garcia-Rodriguez ${ }^{3}$ - Bianca Kollhorst ${ }^{5}$. Pär Karlsson ${ }^{6}$ - Love Linnér ${ }^{6}$. Thomas M. MacDonald ${ }^{4}$. Estel Plana ${ }^{1}$. Ana Ruigómez $z^{3} \cdot$ Tania Schink $^{5} \cdot$ Ryan Ziemiecki $^{2}$ - Elizabeth B. Andrews ${ }^{2}$}

$1 \quad$ RTI Health Solutions, Barcelona, Spain

2 RTI Health Solutions, 3040 East Cornwallis Road, Research Triangle Park, NC 27709, USA

3 Centro Español de Investigación Farmacoepidemiológica, Madrid, Spain

4 University of Dundee, Dundee, Scotland
5 Leibniz Institute for Prevention Research and Epidemiology-BIPS, Bremen, Germany

6 Karolinska Institutet, Stockholm, Sweden

7 Present Address: Department of Cardiology, Hospital Universitari de Bellvitge, Feixa Llarga, L'Hospitalet de Llobregat, Barcelona, Spain 